\title{
Recent breakthroughs in metastatic uveal melanoma: a cause for optimism?
}

\author{
Joseph J Sacco ${ }^{1,2}$, Helen Kalirai ${ }^{1}$, Jenna Kenyani ${ }^{1}$, Carlos R Figueiredo ${ }^{1}$, Judy M Coulson ${ }^{1,3}$ \\ \& Sarah E Coupland*,1,4 \\ ${ }^{1}$ Liverpool Ocular Oncology Research Group, Department of Molecular \& Clinical Cancer Medicine, Institute of Translational \\ Medicine, University of Liverpool, UK \\ ${ }^{2}$ Department of Medical Oncology, Clatterbridge Cancer Centre, Bebington, UK \\ ${ }^{3}$ Department of Cellular \& Molecular Physiology, Institute of Translational Medicine, University of Liverpool, UK \\ ${ }^{4}$ Department of Cellular Pathology, Royal Liverpool University Hospital, Liverpool, UK \\ *Author for correspondence: Tel.: 0151706 5885; s.e.coupland@liverpool.ac.uk
}

\begin{abstract}
"In summary, several promising new agents are in development for UM. Together with a better understanding of the biology of the disease and the recent delivery of relatively large studies, this holds hope that new and more effective treatment options will become available for UM patients with metastatic disease in the near future."
\end{abstract}

First draft submitted: 7 February 2018; Accepted for publication: 21 February 2018; Published online: 9 May 2018

Keywords: checkpoint inhibitors • IMCgp100 • immunotherapy • MEK inhibitors selumetinib • tumor infiltrating lymphocytes • uveal melanoma

Uveal melanoma (UM), a rare subtype of melanoma with a propensity for liver metastasis, remains one of very few malignancies for which there are no proven, effective therapies for metastatic disease. While treatment of primary UM is almost invariably successful, metastatic UM has proven resistant to most therapies assessed thus far; this being compounded by its rarity and consequently a historical lack of well-powered trials. In addition, there has been a tendency to transpose clinical results from cutaneous melanoma (CM) to UM, although it has become clear that while these malignancies share a melanocyte lineage, their biological behavior is very different. However, as we outline below, promising early clinical results for novel drugs and approaches, and an evolving understanding of the basic biology of UM, suggest a more optimistic future for the management of metastatic UM.

Immunotherapy is currently the most exciting area of drug development for cancer, holding out the prospect of durable remissions and even cure for metastatic disease. Unfortunately, although widely used, immunotherapy with established agents (monoclonal antibodies against the immune checkpoints CTLA4 and PD-1) has been disappointing to date in UM. In contrast to CM where response rates to single-agent anti-CTLA4 (ipilimumab) and anti-PD-1 (nivolumab or pembrolizumab) are in the order of 10-20 and 40\%, respectively, in UM response to single-agent therapy with these monoclonal antibodies is generally reported to be in the region of 5\% or less $[1,2]$. The median overall survival in most UM series is less than a year, and there is little evidence to suggest significant disease control, although studies have all been small single-arm and nonrandomized, thus making it difficult to fully assess efficacy. While there has been some enthusiasm for combination CTLA4 and PD-1 blockade (ipilimumab and nivolumab), there remains very little evidence for meaningful clinical activity in UM. A recent study reported a response rate of approximately $17 \%$ in evaluable patients; however the study was limited to 15 patients and median progression-free survival (PFS) was just 2.8 months [2]. Moreover, in a report of a separate expanded access program, which included six patients, none responded and median PFS was similarly 2.8 months [3]. Combined with the considerable morbidity associated with combination immunotherapy, it remains unclear whether this approach is of benefit, and results from ongoing trials are awaited.

The difference in checkpoint inhibitor efficacy between UM and CM is likely to at least partly relate to differences in mutational load and thus cancer cell immunogenicity. A recent study by The Cancer Genome Atlas (TCGA), and others, showed markedly lower mutation rates in UM in comparison to CM or other cancer types [4]. Phenotypically, there is also evidence to suggest that UM is an immunologically 'cold' tumor, and is therefore perhaps not 'primed'

Future $\because$ Medicine 
to respond to checkpoint inhibitors. This is supported by the predominantly peritumoral localization of $\mathrm{CD}^{+}$and $\mathrm{CD}^{+}$T-cell infiltrates in UM metastases, which appear to be excluded from the tumor mass interiors [5], while expression of the PD-1 ligand (PD-L1) is also significantly lower in UM than in CM [6,7].

However, data from the TCGA suggest that this may be an oversimplification. In a proportion of tumors, PD-L1, and more frequently other checkpoint molecules such as IDO1 and TIGIT, are upregulated [4]. In addition, a recent study of adoptive transfer of tumor infiltrating lymphocytes provides direct evidence that tumor specific $\mathrm{T}$ cells are present in UM metastases and that these may be stimulated to engender clinically meaningful responses [8]. This Phase II study involved removal of the metastases, followed by ex vivo expansion of tumor infiltrating lymphocytes, which were infused back into the patient after lymphodepleting conditioning chemotherapy. Results from 21 patients have been reported with seven (35\%) responding, including one complete response that was ongoing at the time of report 21 months after treatment. While this is clearly very early data that will require confirmation (expansion phase ongoing), it does at the very least indicate that UM is not wholly unresponsive to immunotherapy.

An alternative approach to immunotherapy using a novel bispecific molecule, IMCgp 100, has similarly generated much interest. One end of IMCgp100 is a soluble affinity enhanced T-cell receptor targeting the melanocyte lineage protein gp100 bound to HLA A201, while the other end is an anti-CD3 scFv. This allows IMCgp100 to redirect T-cells into UM tumors, leading ultimately to T-cell activation and tumor cell killing. Two Phase I trials have reported initial results in UM; a first in-human dose escalation study that included both UM and CM patients (NCT01211262), and a subsequent study that used an intrapatient dose escalation approach and was limited to UM (NCT2570308). The latter strategy was predicated on an observed amelioration in toxicity after the first few doses and achieved an approximately 50\% increase in the tolerable dose. A pooled analysis of these two studies was presented at SITC in 2017 [9]. While response rates were relatively low (3/15 and 2/19 patients respectively), initial results suggest prolonged disease stabilization in a significant proportion of patients, and most importantly, a 1 year overall survival of $73 \%$ was observed in both studies, which is significantly higher than expected for this population of patients.

Intriguingly, biopsies taken after three doses of IMCgp100 (on day 16) showed an influx of PD- $1^{+} / \mathrm{CD} 8^{+}$ lymphocytes, and an increase in PD-L1 expression in the tumor [9]. In essence, this suggests that IMCgP100 treatment can change a UM tumor from being immunologically 'cold' to 'hot', and in turn that either sequential or combinatorial use of IMCgp100 and checkpoint inhibitors may ultimately improve activity. However, the clinical and biological signals from these two studies will clearly need confirmation in further trials. To this end, both an expansion cohort of the intrapatient dose escalation study (NCT2570308), and a pivotal Phase II study (NCT0307392) randomizing against investigator choice are underway.

While immunotherapy has unsurprisingly been a major focus of attention, there have also been significant advances in our understanding of cell signaling and targeted therapies in UM. Data from the TCGA study and others have confirmed that there are only a limited number of driver mutations in UM [4]. Principle among these are mutations in the genes encoding heterotrimeric g-protein subunits GNAQ and GNA11, one of which is mutated in almost all cases of UM. Notably, the small number of UM without GNAQ/GNA11 mutation in fact harbors mutations in other genes that activate the same pathways: PLCB4 and CYSLTR2. A number of signaling pathways are activated downstream of GNAQ and GNA11, including the MAPK pathway, which has been the principle focus of attention. Preclinical models have shown that UM cell lines are susceptible to inhibition of the MAPK pathway by MEK inhibitors [10], leading in turn to a study of the MEK inhibitor selumetinib in comparison to chemotherapy. This study (NCT01143402) showed improvement in its primary end point of PFS (15.9 vs 7 weeks; $\mathrm{p}<0.001$ ) and response (14 vs $0 \%$ ), but not overall survival (11.8 vs 9.1 months; $\mathrm{p}=0.09$ ), in the selumetinib arm [11]. These results suggest only modest efficacy for selumetinib monotherapy, and subsequent studies have mostly investigated combination with other agents. The SUMIT trial (NCT01974752) was a Phase III study that compared selumetinib or placebo in combination with dacarbazine. This study has not fully reported but did not meet its primary end point of overall survival. However, further trials of MEK inhibitors are underway including a randomized Phase II study (SelPac, ISRCTN 29621851) which is seeking to investigate the activity of selumetinib in combination with the chemotherapy agent paclitaxel in UM, based on evidence that the combination increases induction of apoptosis in preclinical models $[12,13]$. Investigation of agents targeting other components of signaling pathways downstream of GNAQ/GNA11 (such as the PKC inhibitor LXS196, NCT02601378) is also under way.

Other approaches include glembatumumab vedotin, a monoclonal antibody-drug conjugate targeting the glycoprotein 'nonmetastatic b', which is commonly overexpressed in cancers including UM [14]. This has been assessed in a single-arm Phase II study (NCT02363283), which recently reported initial results [15]. The study included 31 
evaluable patients with a reported response rate of $6 \%$, disease control rate of $61 \%$ at 3 months and median overall survival of 11.8 months. While these results suggest the agent may have activity in UM, further investigation is required.

In view of UM's predisposition for metastasis to the liver, and the frequent occurrence of liver only metastases, a number of liver-directed therapies have been investigated over the years. Of these, percutaneous hepatic perfusion with melphalan is perhaps the most studied. An initial randomized Phase III study completed several years ago and showed an improvement in hepatic PFS (7.0 vs 1.6 months; $\mathrm{p}<0.0001)$ when compared with investigators' choice, however no improvement in overall survival was observed (10.6 vs 10.0 months; $\mathrm{p}=0.77)$, although this may have been partly due to patient crossover [16]. More recently, a case series combining data from two major centers reported a median overall survival of 15 months in 51 patients [17]. These results, while encouraging, require confirmation in a randomized study and an international Phase III (FOCUS, NCT026786572) comparing percutaneous hepatic perfusion with investigator choice is currently enrolling patients.

In summary, several promising new agents are in development for UM. Together with a better understanding of the biology of the disease and the recent delivery of relatively large studies, this holds hope that new and more effective treatment options will become available for UM patients with metastatic disease in the near future.

\section{Financial \& competing interests disclosure}

The authors have no relevant affiliations or financial involvement with any organization or entity with a financial interest in or financial conflict with the subject matter or materials discussed in the manuscript. This includes employment, consultancies, honoraria, stock ownership or options, expert testimony, grants or patents received or pending, or royalties.

No writing assistance was utilized in the production of this manuscript.

\section{References}

Papers of special note have been highlighted as: • of interest; $\bullet \bullet$ of considerable interest

1. Heppt MV, Steeb T, Schlager JG et al. Immune checkpoint blockade for unresectable or metastatic uveal melanoma: a systematic review. Cancer Treat. Rev. 60, 44-52 (2017).

- Provides updated clinical outcome data from trials of immune checkpoint inhibitors in patients with uveal melanoma (UM).

2. Heppt MV, Heinzerling L, Kahler KC et al. Prognostic factors and outcomes in metastatic uveal melanoma treated with programmed cell death-1 or combined PD-1/cytotoxic T-lymphocyte antigen-4 inhibition. Eur. J. Cancer 82, 56-65 (2017).

- This systematic review of immune checkpoint blockade in metastatic UM highlights the lack of evidence-based inhibitor selection for UM, which has contributed to the poor response of patients in clinical trials.

3. Shoushtari AN, Navid-Azarbaijani P, Friedman CF et al. Efficacy of nivolumab and ipilimumab (nivo + ipi) combination in melanoma patients (pts) treated at a single institution on an expanded-access program (EAP). J. Clin. Oncol. 34, 9554-9554 (2016).

4. Robertson AG, Shih J, Yau C et al. Integrative analysis identifies four molecular and clinical subsets in uveal melanoma. Cancer Cell 32, 204-220.e215 (2017).

- This work is a landmark study that proposes a new classification model for primary UM patients on the basis of extensive analyses undertaken on phenotypically well-defined UM samples. Differences in the levels of BAP1 loss, DNA methylation, genomic features, immune-related genes and outcomes are disclosed.

5. Krishna Y, McCarthy C, Kalirai H et al. Inflammatory cell infiltrates in advanced metastatic uveal melanoma. Hum. Pathol. 66, 159-166 (2017).

- Describes the density and spatial distribution of tumor-associated macrophages and tumor infiltrating lymphocytes (TILs) within hepatic UM metastasis and shows how in some metastatic UMs, $\mathrm{CD8}^{+}$TILs are located peritumorally, unable to penetrate the tumor mass.

6. Javed A, Arguello D, Johnston C et al. PD-L1 expression in tumor metastasis is different between uveal melanoma and cutaneous melanoma. Immunotherapy 9, 1323-1330 (2017).

7. Qin Y, Petaccia de Macedo M, Reuben A et al. Parallel profiling of immune infiltrate subsets in uveal melanoma versus cutaneous melanoma unveils similarities and differences: a pilot study. Oncoimmunology 6, e1321187 (2017).

8. Chandran SS, Somerville RPT, Yang JC et al. Treatment of metastatic uveal melanoma with adoptive transfer of tumour-infiltrating lymphocytes: a single-centre, two-stage, single-arm, Phase 2 study. Lancet Oncol. 18, 792-802 (2017).

- First report describing an effective adoptive transfer of autologous TILs to mediate objective tumor regression in patients with metastatic UM.

9. Carvajal R, Sato T, Shoushtari A et al. Safety, efficacy and biology of the gp100 TCR-based bispecific T cell redirector, IMCgp100 in advanced uveal melanoma in two Phase 1 trials. J. Immunother. Cancer 5, P208 (2017). 
- Demonstrates the preliminary immune biology, safety and promising efficacy of IMCgp100, a bispecific T-cell redirector for therapy of advanced UM.

10. Ambrosini G, Pratilas CA, Qin LX et al. Identification of unique MEK-dependent genes in GNAQ mutant uveal melanoma involved in cell growth, tumor cell invasion, and MEK resistance. Clin. Cancer Res. 18, 3552-3561 (2012).

- Shows that slumetinib regulates the expression of specific MEK dependent protumorigenic genes in GNAQ mutant cells.

11. Carvajal RD, Sosman JA, Quevedo JF et al. Effect of selumetinib vs chemotherapy on progression-free survival in uveal melanoma: a randomized clinical trial. JAMA 311, 2397-2405 (2014).

12. Holt SV, Logie A, Odedra R et al. The MEK1/2 inhibitor, selumetinib (AZD6244; ARRY-142886), enhances anti-tumour efficacy when combined with conventional chemotherapeutic agents in human tumour xenograft models. Br. J. Cancer 106, 858-866 (2012)

- Discloses the preclinical benefits of inhibiting MEK in combination with several standard chemotherapeutics or an Aurora B kinase inhibitor.

13. MacKeigan JP, Collins TS, Ting JP. MEK inhibition enhances paclitaxel-induced tumor apoptosis. J. Biol. Chem. 275, 38953-38956 (2000).

- First report demonstrating an increase in tumor cell death in response to the combination of paclitaxel with an MEK inhibitor.

14. Williams MD, Esmaeli B, Soheili A et al. GPNMB expression in uveal melanoma: a potential for targeted therapy. Melanoma Res. 20, 184-190 (2010).

15. Patel S, Lewis K, Olencki T et al. A Phase II study of glembatumumab vedotin for metastatic uveal melanoma. Presented at: 14th International Congress of the Society for Melanoma Research. Brisbane, Australia, 18-21 October 2017.

16. Hughes MS, Zager J, Faries $\mathrm{M}$ et al. Results of a randomized controlled multicenter Phase III trial of percutaneous hepatic perfusion compared with best available care for patients with melanoma liver metastases. Ann. Surg. Oncol. 23, 1309-1319 (2016).

17. Karydis I, Gangi A, Wheater MJ et al. Percutaneous hepatic perfusion with melphalan in uveal melanoma: a safe and effective treatment modality in an orphan disease. J. Surg. Oncol. 117, 1170-1178 (2018). 